\title{
Zur Position des „elektrischen“ Erzählers
}

Nicht die Differenz. Sondern das Übereinstimmende ist festzuhalten: Dass die Funktion des Erzählers eine andere sein muss, als noch im Bildungsroman mit seinem Ideal der stabilen, ein für alle Mal traditionell festgelegten Bahnen, wenn von der modernen Welt (und im Bezugsrahmen einer „Dritten Kultur“) überhaupt noch „erzählt“ werden können soll. Denn in dieser unserer modernsten Welt scheint ja der Bahnwechsel keine Ausnahme, sondern die Regel darzustellen. Erstaunlich die Aktualität eines über zehn Jahre alten Literaturtextes, für den sogar die neueste „Krisenschwelgerei“, die der hysterischen Klimawandel-Angst, eben weil sie ungenau auf Apokalyptisches abstellt, ein ,,unnötiges Dilemma“1 bedeutet - demgegenüber, so meint es jedenfalls der Autor Strau $\beta$, „zu Heideggers und Heisenbergs Zeiten“ noch genauer gedacht zu werden vermochte. Selbst wenn man den Erstgenannten nicht uneingeschränkt einbeziehen möchte: Der kulturbeherrschende Zwang, „das politisch Verwertbare an ihren Schlüssen, noch bevor sie tatsächlich gezogen werden dürfen, rasch unter die Leute zu bringen“, weil es ja Fördermittel und Zeitungsplatz zu erobern gelte, ${ }^{2}$ ist gewiss nicht geringer geworden. Das Flaubert'sche Lexikon der Gemeinplätze sollte in Erinnerung behalten werden, fordert der Uckermärker an dieser Stelle - mithin den Rückbezug auf ein zentrales Vorhaben des gleichen Autors, auf den sich Kafka bei seiner Kreierung des Kafkaesken zentral bezogen hatte, wie noch zu zeigen sein wird. Daraus entwickelt sich die womöglich auf- wie

\footnotetext{
${ }^{1}$ Botho Strau $\beta$, Die Unbeholfenen. Bewusstseinsnovelle, a. a. O., S. 59.

${ }^{2}$ Botho Strau $\beta$, Die Unbeholfenen. Bewusstseinsnovelle, a. a. O., S. 60.
}

(C) Der/die Autor(en) 2021

B. Neumann, Umrisse einer Dritten Kultur im interdisziplinären Zusammenspiel zwischen Literatur und Naturwissenschaft, ELECTRISCHER PROMETHEUS. Umrisse einer Dritten Kultur im interdisziplinären Zusammenspiel zwischen Literatur und Naturwissenschaft, https://doi.org/10.1007/978-3-662-63204-8_9 
anregendste These diese Botho Strau $\beta$ 'schen Textes: Dass nämlich die letzte, alles verändernde Bewusstseinskrise, eben die um das ikonische Jahr 1900 herum (deren aktiver Teilnehmer eben auch Franz Kafka gewesen ist), als eine erschienen sei, die derart gründlich in Literatur, Bildender Kunst, Philosophie etc. „gespiegelt“, also reflektiert wurde, dass das heutige Bewusstsein, eben weil es nur auf „Krisen“ abhebt und aus ihnen besteht, selbst gar nicht mehr zur wirklichen Krise fähig sei. Es sei sozusagen eine Impfung erfolgt. Die habe ihren Preis darin besessen, dass mit der Anfälligkeit zugleich auch alle Sensibilität beseitigt worden wäre, radikal und darum anhaltend. Hier nähert Strau $\beta$ sich dem späten Gottfried Benn an: Alles Wesentliche schon gewesen. Hofmannsthal, Vermeer, Proust, Sartre sind passé. Keinerlei Epiphanie scheint mehr möglich, jede Berufung auf die vorgenannten Namen vergeblich. Die „Infodemenz“ sei das Signum unserer Jetztzeit, verfügt Botho Strau $\beta$ abschliessend. Ihr gegenüber sei selbst die höchst entwickelte Geistigkeit, eben die des am „höchsten gebündelten" Geistes, also die des transdisziplinär Kompetenten, auf bedrohtem, wenn nicht gar unrettbar verlorenem Posten. ${ }^{3}$ In den Zeiten der „Arachnotopie“, in denen nur noch das Netz lebt und sich selbstständig fortspinnt, wofür es keine Metapher gäbe; in solchen Zeiten sei denn auch das "schwache Lesen“ entstanden, das nicht aus sich selbst, sondern ,theoriegesteuert“ läse. ${ }^{4}$ Die von einigen wenigen, eben den Strauss'schen „Bündlern“, vorgreifend erreichte „Dritte Kultur“ habe sich nicht wirklich durchgesetzt: „Anders als einen Musil oder Hermann Broch stimuliert offenbar das Wissen seiner Zeit den erzählenden Bewusstseinsmacher nicht mehr“. Der „erzählende Bewusstseinsmacher“ wurde abgelöst von „Belletristen“ und „Sinnpfadfinder“, welche die vormaligen "Symbolsucher“ abgelöst hätten. ${ }^{5}$ „Ich weiss wohl: Das reflektierende Subjekt gehört wie ehedem das sakrale nun schon zum Altertum des Menschengeschlechts.“6 Deshalb seien auch die „Bündler“ (so Strauß' Bezeichnung für die interdisziplinär Interessierten - weil diese die Disziplinen „bündelten“, oder weil sie „Bünde“ wie den, sagen wir, Stefan Georges bevorzugten?), jedenfalls die Pioniere einer „Dritten Kultur“ erschienen existentiell bedroht. Und dies ironischerweise deshalb, kann man an dieser Stelle nur folgern, weil sie in der letzten Moderne um 1900 so durchgehend beherrschend gewesen waren. Eine so

\footnotetext{
${ }^{3}$ Botho Strau $\beta$, Die Unbeholfenen. Bewusstseinsnovelle, a. a. O., S. 63, 64.

${ }^{4}$ Botho Strau $\beta$, Die Unbeholfenen. Bewusstseinsnovelle, a. a. O., S. 84.

${ }^{5}$ Botho Strau $\beta$, Die Unbeholfenen. Bewusstseinsnovelle, a. a. O., S. 85.

${ }^{6}$ Botho Strau $\beta$, Die Unbeholfenen. Bewusstseinsnovelle, a. a. O., S. 86.
} 
apokalyptische wie überraschende Perspektive eröffnet sich: Die ,allermodernste Moderne", wie sie um 1900 herum von Wien ausgegangen sei, die wäre dann die letzte überhaupt gewesen? Strauß glaubt eben dies erkannt zu haben. Die Krisenfähigkeit des menschlichen Bewusstsein überhaupt sei damals ausgeschöpft worden; das heutige „Netz“-Zeitalter sei folglich eines, das solche „Krise“ gar nicht mehr kennen könne. Im finalen Hochschrauben dieses Gedankens: Ein „Bewusstsein“, wie es noch im vergangenen prä-Internet-Zeitalter existiert hätte, könne es gar nicht mehr geben. Undenkbares sei auf diese Weise zur „Realität“ geworden; oder eben doch auch nicht, weil es eine „Realität“ im hergebrachten Sinne ja gar nicht mehr gäbe. Ganz so, wie es nach Adornos berühmt-berüchtigtem Ausspruch niemals ein ,richtiges Leben im falschen“ gibt. Die Sirenen singen nicht mehr. Prometheus ist längst im Anorganischen verschwunden. Bedeutet Existenz also für uns erneut, wie einst in der Frühromantik, ein schreibend betriebenes Sehnen nach einem per definitionem Unerreichbaren? Verkörpert sich darin die Sehnsucht der Lichtstrahlen nach Überschreitung ihrer Möglichkeiten; die Hoffnung des hoffnungslos Heutigen nach dem Gestrigen als eine neue, paradoxe Form von Utopie?

Open Access Dieses Kapitel wird unter der Creative Commons Namensnennung 4.0 International Lizenz (http://creativecommons.org/licenses/by/4.0/deed.de) veröffentlicht, welche die Nutzung, Vervielfältigung, Bearbeitung, Verbreitung und Wiedergabe in jeglichem Medium und Format erlaubt, sofern Sie den/die ursprünglichen Autor(en) und die Quelle ordnungsgemäß nennen, einen Link zur Creative Commons Lizenz beifügen und angeben, ob Änderungen vorgenommen wurden.

Die in diesem Kapitel enthaltenen Bilder und sonstiges Drittmaterial unterliegen ebenfalls der genannten Creative Commons Lizenz, sofern sich aus der Abbildungslegende nichts anderes ergibt. Sofern das betreffende Material nicht unter der genannten Creative Commons Lizenz steht und die betreffende Handlung nicht nach gesetzlichen Vorschriften erlaubt ist, ist für die oben aufgeführten Weiterverwendungen des Materials die Einwilligung des jeweiligen Rechteinhabers einzuholen.

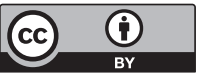

